\title{
PERFIL DE RELAÇÕES INTERPESSOAIS E HABILIDADES SOCIAIS DE ESTUDANTES DE PSICOLOGIA
}

\author{
Wanessa Marques Tavares \\ Psicóloga, mestranda da Universidade Federal de Minas Gerais.
}

Gleiber Couto

Psicólogo, Doutor em Psicologia. Coordenador do Laboratório de Avaliação, Medidas e Instrumentação em Ciências da Saúde - LAMI. Universidade Federal de Goiás, Campus Catalão / UFG-CAC.

\begin{abstract}
Renata Limongi França Coelho Silva
Psicóloga e Pedagoga, Mestre em Psicologia. Professora do Centro de Ensino Superior de Catalão -

\begin{abstract}
Resumo
Este estudo teve como objetivo investigar o perfil de estudantes de Psicologia no que diz respeito às características de suas relações interpessoais e habilidades sociais e compará-lo com outros universitários. Participaram desta pesquisa 153 estudantes de Belo Horizonte e entorno. Os instrumentos utilizados foram o Checklist de Relações Interpessoais-II (CLOIT-II) e o Inventário de Habilidades Sociais (IHS). Foram traçados os perfis de resposta e estimada a significância das diferenças por meio do teste $t$ de Student. Os resultados indicaram que o perfil dos estudantes de Psicologia pode ser caracterizado por relações mais amigáveis, maior disposição para as interações e menor demonstração de afeto, além de possuírem mais habilidades sociais que os demais universitários. Assim, pôde-se verificar que os estudantes de Psicologia percebem suas relações interpessoais como sendo adaptativas aos padrões sociais e suas habilidades sociais são um pouco mais expressivas que as de universitários de outros cursos superiores.
\end{abstract}

Palavras-chave: universitários; psicologia, perfil psicológico.

\section{PROFILE OF INTERPESONAL RELATIONS AND SOCIAL SKILLS OF PSYCHOLOGY UNDERGRADUATES}

\begin{abstract}
This study the profile of Psychology undergraduates was compared to that of other undergraduates. Interpersonal relations and social skills were evaluated using the Checklist for Interpersonal Transactions-II (CLOIT-II) and the Social Skills Inventory (IHS). 153 students were studied in Belo Horizonte and surroundings. Significance of profile difference was evaluated using the Student's t-test. The results suggest that the profile of Psychology undergraduates can be characterized by more friendly relations, greater willingness to interact, showing less affection and having more social skills than other undergraduates. This lead to the conclusion that Psychology undergraduates perceive their interpersonal relations as adaptive to social models and their social skills as a little more apparent than undergraduates in other areas of studies.
\end{abstract}

Keywords: undergraduates, psychology, psychological profile. 


\title{
PERFIL DE LAS RELACIONES INTERPESONALES Y LAS HABILIDADES SOCIALES PARA ESTUDIANTES DE PSICOLOGÍA
}

\begin{abstract}
Resumen
En este estudio el perfil de los estudiantes de Psicología se comparó con la de otros estudiantes. Se evaluaron las relaciones interpersonales y las habilidades sociales utilizando la Cheicklist for Interpersonal Transactions-II (CLOIT-II) y el Social Skills Inventory (IHS). Los sujetos fueron 153 estudiantes de Belo Horizonte y sus alrededores. Significación de la diferencia perfil se evaluó mediante la Student's ttest. Los resultados sugieren que el perfil de los estudiantes de Psicología se caracteriza por unas relaciones más amistosas, una mayor disposición a interactuar, muestran menos afecto y tener más habilidades sociales que los otros estudiantes. Esto llevó a la conclusión de que estudiantes de Psicología perciben sus relaciones interpersonales, adaptación a los modelos sociales y sus habilidades sociales como un poco más aparente que estudiantes en otras áreas.
\end{abstract}

Palabras clave: estudiantes, psicología, perfil psicológico.

\section{INTRODUÇÃO}

Desde os anos 60 já são encontrados na literatura especializada registros acerca de estudos sobre habilidades sociais. Nesta época, os ingleses se interessaram pelo tema, tomando por base estudos ligados à Psicologia Social e das Organizações, disseminando assim, o termo habilidades sociais (Argyle \& Kendon, 1967). Alguns anos mais tarde, Lazarus (1973) propôs quatro tipos de respostas socialmente hábeis, a saber, a capacidade de dizer não; de expressar sentimentos positivos e negativos; de pedir favores e fazer pedidos; e de iniciar, manter e encerrar diálogos. A partir daí, outras categorias de resposta foram sugeridas, como aceitar pedidos, fazer elogios e falar em público (Rathus, 1975). Na década de 1980, o estudo acerca do Treinamento de Habilidades Sociais se desenvolveu plenamente, demonstrando resultados positivos sobre dificuldades de natureza interpessoal, como, por exemplo, habilidades de comunicação e resolução de problemas (Wolp, 1976).

Um indivíduo pode ser considerado socialmente habilidoso quando seu comportamento apresenta-se apropriado, ou seja, é caracterizado por ações eficazes e não-danosas na especificidade de diversas situações (Caballo, 1996; 2003). De maneira geral, as habilidades sociais representam o conjunto de comportamentos que permitem o desempenho social ajustado, sendo este expresso pelo padrão de relações interpessoais que o indivíduo possui. Tal desempenho é avaliado pela competência social, que se revela pela capacidade de um indivíduo se engajar em situações interpessoais de forma a alcançar os 
objetivos da interação, mantendo o equilíbrio de poder e as trocas positivas na relação com as outras pessoas na interação. Desta forma, as interações interpessoais podem ser caracterizadas conforme sua capacidade de produzir os efeitos pessoais e sociais desejados, ou seja, nos termos da satisfação das necessidades individuais (Del Prette \& Del Prette, 1999, 2001a).

Kiesler (1983) elaborou um modelo para representar os diferentes tipos de relações interpessoais, o Círculo Interpessoal, baseado no modelo circumplexo. Uma característica distintiva deste modelo é que a matriz de correlações, entre um conjunto de variáveis, apresenta valores em que um grupo de correlações aumenta na mesma proporção que outro grupo diminui. Quando as variáveis são posicionadas em um plano bidimensional, se apresentam no formato de círculo. Essa ordem circular permite uma representação geométrica de variáveis qualitativamente distintas numa sequência determinada por grau de correlação, sem Ihes impor uma hierarquia (Fischer, 1996). O Círculo Interpessoal de Kiesler é constituído por 16 posições interpessoais, que são diferentes misturas de Dominância-Submissão e de Amigabilidade-Hostilidade. Suas categorias são rotuladas pelas letras de $\mathrm{A}$ a $\mathrm{P}$ e distribuídas ao redor da circunferência em um sentido anti-horário (Kiesler, 1983).

Tendo em vista que o conjunto de habilidades sociais que o indivíduo possui vai refletir diretamente a qualidade das suas relações interpessoais, e consequentemente, impactar a sua vida pessoal, profissional e social (Del Prette \& Del Prette, 1999, 2001a), algumas pesquisas foram realizadas com o intuito de investigar quais variáveis estão relacionadas com um perfil deficitário ou adequado de habilidades sociais. Por exemplo, foram encontradas pesquisas que investigaram a relação entre pais e filhos e sua influência no desenvolvimento de tais habilidades. É o caso de Eisenberg et al (1993), que desenvolveram um estudo com o objetivo de investigar a relação entre as habilidades sociais paternas e o comportamento dos filhos. Os resultados apontaram que pais socialmente habilidosos tendem a exercer a monitoria positiva e o comportamento moral como estratégias educacionais, evitando que seus filhos desenvolvam comportamentos anti-sociais. Em uma pesquisa similar, Gomide, Salvo, Pinheiro e Sabbag (2005) descobriram que a maioria dos membros das famílias que apresentam práticas educativas que colaboram para o desenvolvimento dos filhos, obteve índice de habilidades sociais elevado, ao contrário das famílias que possuem práticas que prejudicam a evolução dos 
filhos. Da mesma forma, Cia, Pamplin e Del Prette (2006) compararam indicadores de envolvimento de pais e filhos com o repertório de habilidades das crianças. As mães apresentaram, conforme a avaliação dos filhos, melhores indicadores de comunicação e participação que os pais, o que correlacionou positivamente com os escores de habilidades sociais das crianças.

Outros pesquisadores, como Carneiro, Falcone, Clark, Del Prette e Del Prette (2007), investigaram a relação entre qualidade de vida e habilidades sociais, em um estudo com idosos. Os autores verificaram que o grupo de idosos residentes em asilo apresentou um menor repertório de habilidades sociais, uma menor rede de apoio social, maior prevalência de depressão e uma pior qualidade de vida que os que não vivam em asilos. O estudo concluiu que um repertório positivo dessas habilidades é importante para a qualidade de vida e saúde dos idosos. As habilidades sociais também foram investigadas em quadros clínicos, como por exemplo, na esquizofrenia. Foi encontrado que as habilidades sociais foram significativamente prejudicadas nos indivíduos com histórico profissional ruim em relação aos pacientes com um bom histórico (Dickinson, Bellack \& Gold, 2007). Outra variável importante no estudo das habilidades sociais são as questões sociodemográficas. Kliewer (1991) descobriu que a criança vai ampliando suas habilidades ao ser inserida em novos contextos sociais que exigem demandas diferenciadas de desempenho, o que também foi demonstrado por Bandeira, Rocha, Freitas, Del Prette e Del Prette (2006), que descobriram que quanto maior a escolaridade dos pais e seu nível socioeconômico, maior o escore das habilidades sociais, e quanto menor a importância atribuída pelos pais às habilidades, menores os escores das crianças.

Estes são alguns exemplos de trabalhos envolvendo a relação entre as habilidades sociais e outras características pessoais ou sociais. Têm-se observado também um grande interesse dos estudiosos em mapear o comportamento dos universitários, devido às peculiaridades desta fase, em que os estudantes precisam elaborar os conflitos profissionais que surgem e se prepararem para enfrentar o mercado de trabalho (Bueno, Lemos \& Tomé, 2004; Carmo, 2011), por isso esta configura-se uma das etapas que mais exigem comportamentos socialmente habilidosos (Del Prette \& Del Prette, 2004; Costa et al, 2009; Bolsoni-Silva, 2009). Del Prette e Del Prette (1983) afirmam ainda que a formação de terceiro grau deveria incluir em seus objetivos acadêmicos o desenvolvimento interpessoal dos graduandos. Campos, Silva-Filho, Campos e 
Rocha (1996) também salientam a importância de estudos sobre as características de universitários como forma de subsidiar planejamentos pedagógicos e tomadas de decisão mais adequados.

Dessa forma, alguns pesquisadores investigaram as habilidades sociais de estudantes universitários. É o caso de Bueno, Oliveira e Oliveira (2001), que desenvolveram um estudo correlacional entre habilidades sociais e traços de personalidade com estudantes de vários cursos superiores. Seus resultados apontaram para diferenças estatisticamente significativas entre gêneros nas habilidades de enfrentamento com risco e auto-afirmação na expressão de afetos positivos. O estudo também encontrou evidências em relação à associação de diferentes traços de personalidade com diferentes habilidades sociais. Bartholomeu, Nunes e Machado (2008) realizaram uma pesquisa similar, analisando as relações entre habilidades sociais e traços de personalidade englobados por socialização em universitários. Foram encontradas correlações significativas entre amabilidade, auto-afirmação e todos os fatores do IHS. Por outro lado, Bolsoni-Silva, Loureiro, Rosa e Oliveira (2010) se propuseram a descrever as habilidades sociais apresentadas por universitários ao longo dos anos de graduação, investigando mudanças com o passar dos períodos letivos. Os resultados indicaram diferenças de gênero e entre os períodos integral e noturno. As autoras concluíram que os dois primeiros anos na universidade podem ser considerados o período em que os alunos mais necessitam de intervenções, pois apresentam maiores dificuldades para resolver conflitos e se comunicar com os outros. Já Riggio, Watring e Throckmorton (1993) examinaram as relações entre uma medida padronizada de habilidades sociais e o relato de questões relacionadas com o ajustamento psicossocial de 136 universitários. Foi encontrado que habilidades sociais positivas correlacionaram com percepção de apoio social, satisfação com a faculdade e satisfação com a vida em geral.

Dentre as pesquisas que investigam as habilidades sociais de universitários, é frequente a caracterização dos estudantes de psicologia. Um exemplo é o estudo de Del Prette, Del Prette e Castelo Branco (1992), que avaliaram as dimensões do repertório de competência social, conceito que engloba as habilidades sociais, de alunos de início e término do curso de Psicologia. Os resultados indicaram um amplo padrão de semelhanças entre o repertório social do aluno de início e término do curso. Em um estudo parecido, 
Del Prette et al (2004) buscaram caracterizar o repertório de habilidades sociais dos universitários do curso de Psicologia, utilizando uma amostra de quatro estados (MG, BA, RJ e SP). Os resultados indicaram que os estudantes de psicologia apresentaram escores mais altos que os da amostra normativa no escore geral e nos fatores Enfrentamento e auto-afirmação com risco, Conversação e desenvoltura social e Auto-exposição a desconhecidos e situações novas e inferiores nos fatores Auto-afirmação na expressão de afeto positivo e Autocontrole da agressividade em situações aversivas. Por outro lado, as diferenças de sexo seguiram os padrões normativos. Os resultados apontaram também que houve influência da localidade, sexo e idade sobre os escores, com interação local-sexo e local-idade. Os autores concluíram que os dados encontrados fornecem indicadores de validade pra o IHS, pois mostraram um amplo padrão de semelhanças, com algumas diferenças somente em aspectos pontuais. Em uma pesquisa similar, Bolsoni-Silva (2009) comparou as habilidades sociais de estudantes de psicologia antes e após frenquentarem um estágio supervisionado em treinamento de habilidades sociais. Os resultados mostraram melhoras em quatro dos cinco fatores avaliados. A autora concluiu que a supervisão foi um ambiente que propiciou a generalização das habilidades sociais.

Tendo em vista as relações encontradas na literatura entre habilidades sociais e outras características psicológicas, pôde-se verificar sua importância para explicar determinados padrões de relacionamento interpessoal. Pôde-se observar também que o momento de vida no qual as pessoas se encontram e suas escolhas educacionais e profissionais dizem muito a respeito de suas características pessoais e comportamentos, como é visto nos estudos com universitários. Desta forma, o objetivo deste trabalho foi investigar o perfil dos estudantes de psicologia no que diz respeito às características de suas relações interpessoais e habilidades sociais e compará-lo com universitários de outros cursos que compuseram a amostra do estudo de Bueno, Oliveira e Oliveira (2001), no caso das habilidades sociais, e com os sujeitos da amostra de padronização brasileira do Checklist de Relações Interpessoais-II (CLOIT-II), no caso das relações interpessoais. 


\section{MÉTODO}

Participantes

Participaram desta pesquisa um grupo de 153 estudantes de Belo Horizonte e entorno, com idades variando entre 19 e 60 anos $(M=22,67 ; D P=7,41)$, sendo $80,4 \%$ de sujeitos do sexo feminino. Todos os participantes cursavam Psicologia, destes, 3,9\% já possuíam outro curso superior.

Instrumentos

Checklist de Relações Interpessoais-II (CLOIT-II)

Trata-se de um inventário construído com a finalidade de mapear o comportamento interpessoal de pessoas alvo. É apresentado em três formas: Auto-classificação, Transator e Observador. Cada uma delas deve ser respondida pela Pessoa Alvo, por uma pessoa que interage com ela, também chamada de transator, e por um observador que presencia as interações da Pessoa Alvo, respectivamente. Foi traduzido e adaptado por Couto, Van Hattum, Vandenberghe e Benfica (2005) e teve suas propriedades psicométricas e aplicações para a população brasileira investigadas por Couto, Vandenberghe, Van Hattum e Campos (2006) e Couto, Muniz, Vandenberghe e Van Hattum (2008). Neste estudo foi utilizada a forma de auto classificação, que deve ser respondida pela pessoa alvo, e contém 96 proposições que descrevem ações que podem ocorrer em interações entre pessoas.

As proposições estão divididas em 16 escalas bi-dimensionais rotuladas pela letra de $\mathrm{A}$ a $\mathrm{P}$ e distribuídas ao redor de um modelo circular em um sentido antihorário, a saber, Dominância (A), Competição (B), Desconfiança (C), Frieza Afetiva (D), Hostilidade (E), Isolamento (F), Inibição (G), Insegurança (H), Submissão (I), Deferência (J), Confiança (K), Calor Afetivo (L), Amigabilidade $(\mathrm{M})$, Sociabilidade $(\mathrm{N})$, Exposição $(\mathrm{O})$ e Segurança $(\mathrm{P})$. Cada escala pode receber um escore bruto que varia entre 0 e 9 pontos. Os escores nas 16 escalas podem ser combinados com o objetivo de descrever padrões mais complexos de comportamentos. Por exemplo, os quadrantes são escalas combinadas pela soma dos componentes trigonométricos das escalas que compõe cada quarto do círculo. As fórmulas são: Hostilidade-Dominância (Qdt-HD) $=0,707 \mathrm{~A}+0,924 \mathrm{~B}$ $+\mathrm{C}+0,924 \mathrm{D}+0,707 \mathrm{E} ;$ Hostilidade-Submissão $(\mathrm{Qdt}-\mathrm{HS})=0,707 \mathrm{E}+0,924 \mathrm{~F}$ 
$+\mathrm{G}+$ 0,924 H + 0,707 I; Amigabilidade-Submissão (Qdt-AS) $=0,707 \mathrm{I}+0,924$ $\mathrm{J}+\mathrm{K}+0,924 \mathrm{~L}+0,707 \mathrm{M}$; Amigabilidade-Dominância $(\mathrm{Qdt}-\mathrm{AD})=0,707 \mathrm{M}+$ $0,924 \mathrm{~N}+\mathrm{O}+0,924 \mathrm{P}+0,707 \mathrm{~A}$. Também os escores nas 16 escalas podem ser somados para obter escores separados para os quatro hemisférios possíveis do círculo que descrevem padrões típicos de comportamentos, são eles, Dominância (DOM), Submissão (SUB), Amigabilidade (AMI) e Hostilidade (HOS). As fórmulas para compor os escores dos hemisférios são: Dominância (HmfDOM $)=0,383 \mathrm{~N}+0,707 \mathrm{O}+0,924 \mathrm{P}+\mathrm{A}+0,924 \mathrm{~B}+0,707 \mathrm{C}+0,383 \mathrm{D}$; Submissão $(\mathrm{Hmf}-\mathrm{SUB})=0,383 \mathrm{~F}+0,707 \mathrm{G}+0,924 \mathrm{H}+\mathrm{I}+0,924 \mathrm{~J}+0,707 \mathrm{~K}$ $+0,383 \mathrm{~L}$; Amigabilidade (Hmf-AMI) $=0,383 \mathrm{~J}+0,707 \mathrm{~K}+0,924 \mathrm{~L}+\mathrm{M}+$ $0,924 \mathrm{~N}+0,707 \mathrm{O}+0,383 \mathrm{P} ;$ Hostilidade $(\mathrm{Hmf}-\mathrm{HOS})=0,383 \mathrm{~B}+0,707 \mathrm{C}+$ $0,924 \mathrm{D}+\mathrm{E}+0,924 \mathrm{~F}+0,707 \mathrm{G}+0,383 \mathrm{H}$. Além disso, são providenciados escores que representam o vetor geral da Pessoa Alvo em cada eixo do círculo, usando o peso trigonométrico dos escores de um determinado protocolo. $O$ escore no Eixo vertical interpretado como Controle, pode ser obtido pela fórmula: CONTROLE $($ EX-CON $)=\mathrm{A}-\mathrm{I}+0,924(\mathrm{~B}+\mathrm{P}-\mathrm{H}-\mathrm{J})+0,707(\mathrm{C}+\mathrm{O}-\mathrm{G}-\mathrm{K})+$ $0,383(D+N-F-L)$ e o escore no eixo horizontal interpretado como Filia pode ser obtido pela seguinte fórmula: FILIA $(E x-F I L)=M-E+0,924(N+L-D-F)$ $+0,707(O+K-C-G)+0,383(P+J-B-H)$ (Kiesler, Goldston \& Schmidt, 1991).

Inventário de Habilidades Sociais (IHS) (Del Prette \& Del Prette, 2001b)

Composto por 38 itens que avaliam o repertório de habilidades sociais, baseado na estimativa que o respondente faz sobre a frequência com que reage da forma indicada em cada item, considerando um total de dez vezes em que se encontrou naquela situação e marcando sua resposta com base em uma escala tipo Likert de cinco pontos, que varia de nunca/raramente ( 0 a $20 \%$ das vezes) a sempre/quase sempre ( 81 a $100 \%$ das vezes). Alguns itens são pontuados de forma invertida para a obtenção do escore, pois o modo como são descritos indica que uma frequência alta sugere um déficit na habilidade solicitada naquela situação. Os itens estão organizados em cinco fatores, a saber, Enfrentamento e Auto-afirmação com Risco; Auto-afirmação na expressão de afeto positivo; Conversação e Desenvoltura Social; Auto-exposição a Desconhecidos e a Situações Novas e Autocontrole da agressividade em Situações Aversivas. 
Quanto às propriedades psicométricas, a escala total do instrumento apresenta um índice alfa de Cronbach de 0,75, variando nos fatores entre 0,74 e 0,97. A correlação entre o escore geral e os fatores, bem como entre os quatro primeiros escores fatoriais do IHS é altamente significativa $(p<0.01)$ (Del Prette \& Del Prette, 2001b).

Procedimento de coleta de dados

A pesquisa acadêmica científica pauta-se sobre determinados princípios éticos, que visam assegurar a proteção do direito, bem-estar e dignidade dos participantes, bem como a finalidade e veracidade dos dados obtidos. No presente caso, buscou-se manter os requisitos de participação optativa e anonimato, assim como manter o comprometimento da não manipulação dos dados. Primeiramente, a pesquisa foi avaliada e aprovada pelo Comitê de Ética da Universidade Federal Goiás. A coleta de dados ocorreu de forma coletiva nas salas de aula. Os alunos foram convidados a participar da pesquisa, esclarecidos quanto aos objetivos e procedimentos e ao caráter voluntário de sua participação. Aqueles que concordaram em participar, assinaram o Termo de Consentimento Livre e Esclarecido (TCLE) e foram orientados a responder ao teste CLOIT-II. Em seguida, os alunos responderam o IHS.

\section{Análise de dados}

Ao final das aplicações, os testes foram corrigidos e os resultados dos protocolos foram tabulados em planilha eletrônica. Foram estimadas as estatísticas descritivas das escalas de ambos os testes, comparadas com a amostra do estudo de Bueno, Oliveira e Oliveira (2001), no caso do IHS, e com a amostra de padronização brasileira do CLOIT-II, no caso deste instrumento. Por fim, foi estimada a significância das diferenças por meio do teste $t$ de Student.

\section{RESULTADOS E DISCUSSÃO}

A Figura 1 mostra a comparação de médias das escalas entre os estudantes de psicologia e os sujeitos da amostra de padronização brasileira do CLOIT-II. 


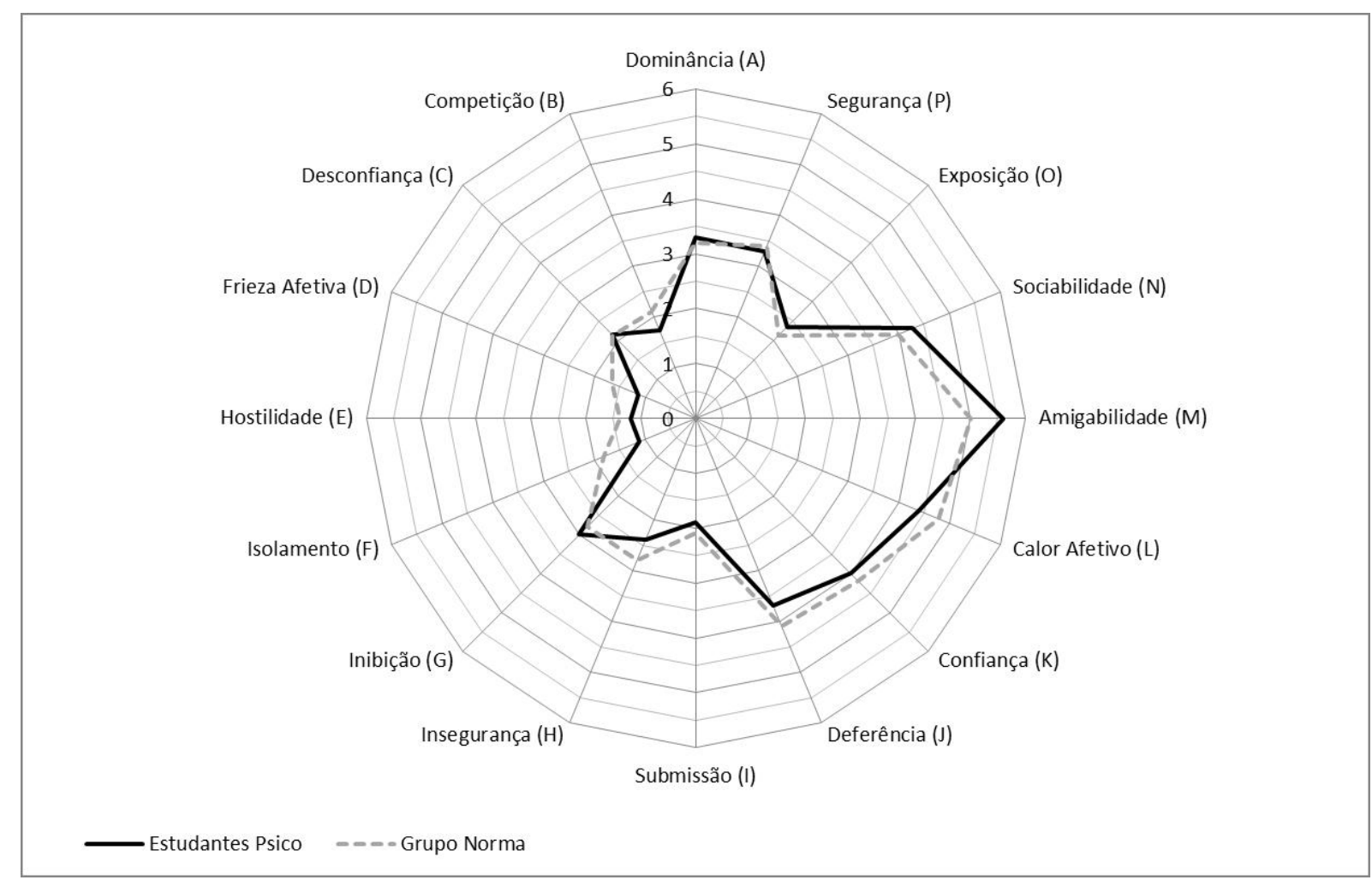

Figura 1.

Comparação de médias entre o grupo normativo e grupo de estudantes de psicologia.

Os resultados indicaram que os padrões mais percebidos nas interações interpessoais entre os estudantes de psicologia foram Amigabilidade, Calor Afetivo e Sociabilidade. No que se refere à característica de sociabilidade, podese observar certa semelhança com um dos resultados encontrados no estudo de interesses profissionais feito por Bueno, Lemos e Tomé (2004), o qual apontou que os alunos do curso de Psicologia apresentaram alto interesse em atividades sociais. Os indivíduos da amostra normativa também percebem suas interações interpessoais baseadas em Amigabilidade e Calor Afetivo, porém, o terceiro padrão mais escolhido para descrever suas interações interpessoais foi Confiança. Contudo, os resultados do $t$-test indicaram uma diferença significativa entre as amostras apenas em Amigabilidade $[T(1,152)=3,00 ; p<0,001]$, com maiores escores a favor dos estudantes de psicologia, e Calor Afetivo $[T(1,152)=-2,25 ; p<0,02]$, com maiores escores a favor dos indivíduos da amostra normativa. Desta forma, os resultados permitem interpretar que os estudantes de psicologia percebem suas interações interpessoais como mais amigáveis e menos calorosas que os universitários em geral. Carmo (2011) descobriu que o universitário de psicologia que atinge um equilíbrio afetivo maior 
tende a se apropriar de seu processo de formação de forma mais madura, o que pode estar relacionado com o fato da característica de calor afetivo ser pouco presente nesse aluno.

Observando os padrões que menos descrevem as interações interpessoais, percebe-se que os estudantes de psicologia apresentaram a menor pontuação em Isolamento, Frieza Afetiva e Hostilidade, assim como a amostra normativa, entretanto as diferenças foram significativas somente nas escalas Isolamento $[T(1,152)=-5,00 ; p<0,001]$ e Frieza Afetiva $[T(1,152)=-4,70 ; p<0,001]$, com menores escores a favor dos estudantes de psicologia. Tais resultados apontam que os estudantes de psicologia se percebem menos isolados e frios na demonstração de afeto em suas relações do que os alunos da amostra normativa.

A Tabela 1 apresenta a comparação de médias dos quadrantes, hemisférios e eixos entre os estudantes de psicologia e o grupo normativo.

Tabela 1.

Médias dos quadrantes, hemisférios e eixos do grupo normativo e dos estudantes de psicologia.

\begin{tabular}{cccccc}
\hline & & \multicolumn{2}{c}{ Médias } & \multicolumn{2}{c}{ Teste $t$} \\
\hline \multirow{5}{*}{ Quadrantes } & Grupo & Estudantes & $t$ & $p$ \\
& Normativo & de Psicologia & & \\
& HOS-DOM & 8,62 & 7,97 & 1,76 & 0,08 \\
& HOS-SUB & 9,38 & 8,55 & 1,88 & 0,05 \\
& AMI-SUB & 17,32 & 16,93 & 0,75 & 0,45 \\
& AMI-DOM & 14,84 & 15,62 & 1,57 & 0,11 \\
\hline Hemisférios & DOM & 13,36 & 13,19 & 0,33 & 0,74 \\
& SUB & 15,81 & 14,83 & 1,77 & 0,07 \\
& AMI & 20,49 & 20,83 & 0,59 & 0,34 \\
& HOS & 9,71 & 8,53 & 2,63 & 0,01 \\
\hline Eixos & CONTROLE & $-2,45$ & $-1,63$ & 1,33 & 0,18 \\
& FILIA & 10,78 & 12,30 & 2,69 & 0,01 \\
\hline
\end{tabular}

Os resultados indicaram que a diferença foi significativa $(p<0,05)$ no quadrante Hostilidade-Submissão, ou seja, o grupo normativo apresenta mais padrões de interação baseados em hostilidade e submissão do que os estudantes de psicologia. Quando observadas as escalas que compõe o quadrante Hostilidade-Submissão separadamente, a saber, Hostilidade, Isolamento, Inibição, Insegurança e Submissão, nota-se que apenas a escala Isolamento 
possui diferença estatisticamente significativa, mas quando analisadas em conjunto, a diferença entre os dois grupos passa a ser significativa.

Em relação aos hemisférios, pode-se observar que somente o hemisfério Hostilidade apresentou diferença significativa ao nível de 0,01, o que confirma o resultado dos quadrantes, o fato dos estudantes do grupo normativo apresentarem comportamentos mais hostis que os estudantes de psicologia. De forma similar, o eixo Filia também apresenta diferença de médias significativa e mostra que os universitários de psicologia apresentam padrões de interação mais amigáveis que o grupo normativo. Carmo (2011), em um estudo sobre afetividade em universitários de psicologia, encontrou que uma das dificuldades relatadas pelos alunos durante a graduação é o fato de estarem sempre sendo cobrados de manter uma postura amigável e bondosa.

A Tabela 2 mostra as estatísticas descritivas da pesquisa de Bueno, Oliveira e Oliveira (2001) e do presente estudo.

Tabela 2.

Estatísticas descritivas Bueno, Oliveira e Oliveira (2001) e estudantes de psicologia.

\begin{tabular}{|c|c|c|c|c|c|c|}
\hline \multirow[b]{2}{*}{ Fatores } & \multicolumn{3}{|c|}{$\begin{array}{c}\text { Amostra do estudo Bueno } \\
\text { et al. }\end{array}$} & \multicolumn{3}{|c|}{ Estudantes de Psicologia } \\
\hline & $\mathrm{N}$ & Média & DP & $\mathrm{N}$ & Média & DP \\
\hline Enfrentamento & 189 & 23,08 & 7,55 & 153 & 22,67 & 7,41 \\
\hline Sentimento Positivo & 189 & 19,25 & 3,89 & 153 & 21,79 & 3,61 \\
\hline Conversação & 189 & 17,73 & 4,43 & 153 & 18,80 & 4,36 \\
\hline Situações Novas & 189 & 7,41 & 2,66 & 153 & 9,78 & 3,48 \\
\hline Agressividade & 189 & 6,92 & 1,88 & 153 & 8,66 & 2,11 \\
\hline N Válido & 189 & & & 153 & & \\
\hline
\end{tabular}

Os resultados apresentados na Tabela 2 mostram que os estudantes de psicologia descrevem habilidades sociais em um maior número de situações que os outros universitários da amostra estudada por Bueno, Oliveira e Oliveira (2001), exceto no fator Enfrentamento e Auto-afirmação com Risco. Ao notar as diferenças das médias, foi feita uma estimativa de significância por meio do teste $t$ de Student.

No fator Enfrentamento e Auto-afirmação com risco, a diferença não foi significativa, ou seja, a probabilidade de ocorrência ao acaso não deve ser tolerada. Nos demais fatores, Auto-afirmação na expressão de sentimento positivo $[T(1,152)=8,70 ; \quad p<0,001]$, Conversação e Desenvoltura Social 
$[T(1,152)=3,04 ; p<0,001]$, Auto-exposição a desconhecidos/situações novas $[T(1,152)=8,42 ; p<0,001]$ e Autocontrole da Agressividade $[T(1,152)=10,23$; $p<0,001]$, os resultados do $t$-test mostraram que as diferenças foram significativas, logo os estudantes de psicologia apresentam relato de desenvoltura social em um número maior de situações sociais. Este resultado corrobora a pesquisa feita por Del Prette, Del Prette e Castelo Branco (1992), na qual encontraram altas porcentagens de relatos de emissão da resposta indicadora de competência social nos alunos do curso de Psicologia. Já os resultados referentes aos fatores Conversação e desenvoltura social e Autoexposição a desconhecidos e situações novas corroboram com os achados da pesquisa de Del Prette et al (2004), na qual os universitários do curso de Psicologia apresentaram escores mais altos que os da amostra normativa nestes fatores.

\section{CONSIDERAÇÕES FINAIS}

O objetivo deste trabalho foi investigar o perfil dos estudantes de psicologia no que diz respeito às características de suas relações interpessoais e habilidades sociais e compará-lo com outros universitários. Sendo assim, os resultados encontrados indicaram que o perfil dos alunos de psicologia, no concernente às relações interpessoais, foi caracterizado por relações mais amigáveis e maior disposição para as interações que os estudantes de outros cursos, além de uma menor presença de demonstração de afeto, tanto positivo quanto negativo. Ao serem observadas as habilidades sociais, os alunos de psicologia se apresentaram como mais habilidosos que outros universitários na maioria das situações descritas. Deste modo, pôde-se verificar que os estudantes de psicologia percebem suas relações interpessoais como sendo adaptativas aos padrões sociais e suas habilidades sociais são um pouco mais expressivas que as de universitários de outros cursos superiores.

Vale ressaltar que as diferenças de médias entre os estudantes de psicologia e os demais universitários foram sutis. Sendo assim, uma hipótese para explicar os resultados encontrados pode estar na formação acadêmica. Pôde-se notar que os alunos de psicologia caracterizam suas relações interpessoais baseadas predominantemente em amizade e amabilidade. Estas características são citadas frequentemente durante a formação na graduação em Psicologia como sendo importantes para o exercício da profissão (Costa et al, 
2008), o que pode exercer uma pressão, mesmo que de forma indireta, para que os alunos desde já se sintam assim, colaborando para a distorção de sua autoimagem ao se descreverem. O fato de serem mais abertos para as interações e possuírem mais habilidades sociais levanta outra hipótese, a de que isto também se deve à cobrança constante de tais comportamentos durante a graduação. Del Prette, Del Prette e Castelo Branco (1992) e Bastos e Achcar (1994) apontam que, na formação do psicólogo, há uma forte cobrança de desenvolvimento da competência social, pois esta pode ser considerada como um dos objetivos da própria atuação profissional do psicólogo, visto que o mesmo é um mediador na promoção de relações sociais mais equilibradas (Del Prette, 1986). Segundo Caballo (1996), as pessoas passam a maior parte do seu tempo, tanto no contexto profissional como pessoal, envolvidas em interações sociais, e estas configuram um dos principais instrumentos de atuação do psicólogo. Sendo assim, na universidade, o professor ensina não somente comportamentos ligados diretamente à prática profissional, mas também estimula a aquisição de comportamentos que apontem para interações sociais mais satisfatórias, pois são repertórios exigidos pela prática acadêmica e importantes para a saúde mental e para a futura vida profissional dos estudantes de psicologia. No que diz respeito à dificuldade de expressar afeto, também pode ser produto da formação, pois a ideia de que o psicólogo tem que aprender a não se envolver demasiadamente com as questões trazidas pelo paciente ou pelo grupo e deve desenvolver uma flexibilidade intelectual para lidar com as mais diversas situações é recorrente nas teorias psicológicas e nas falas dos professores durante o curso (Bastos \& Achcar, 1994; Bolsoni-Silva, 2009).

Embora os resultados desta pesquisa tenham permitido compreender as diferenças entre os estudantes de psicologia da amostra estudada e os universitários de outros cursos, considerando a amostra para padronização do CLOIT-II e amostra do estudo de Bueno, Oliveira e Oliveira (2001), no que diz respeito às habilidades sociais e relações interpessoais, recomenda-se a realização de outros estudos com amostras maiores em outras regiões do país, a fim de verificar se o perfil dos alunos de psicologia realmente se difere dos demais cursos de graduação, se as hipóteses levantas pelo presente estudo podem ser confirmadas ou refutadas e se os resultados encontrados podem ser generalizados. 


\section{REFERÊNCIAS}

Bastos, A. V. B., \& Achcar, R. (1994). Dinâmica profissional e formação do psicólogo: uma perspectiva de integração. In: R. Achcar, A. P. Duran \& A. V. B. Bastos. Psicólogo brasileiro: Práticas emergentes e desafios para a formação (pp. 299-330). São Paulo: Casa do Psicólogo.

Argyle, M. \& Kendon, A. (1967). The experimental analysis of social performance. Advances in Experimental Social Psychology, 3, 55-98.

Bandeira, M., Rocha, S. S., Freitas, L. C., Del Prette, Z. A. P., \& Del Prette, A. (2006). Habilidades sociais e variáveis sociodemográficas em estudantes do ensino fundamental. Psicologia em Estudo, 11(3), 541-549.

Bartholomeu, D., Nunes, C. H. S. S., \& Machado, A. A. (2008). Traços de personalidade e habilidades sociais em universitários. PsicoUSF, 13(1), 4150.

Bolsoni-Silva, A. T. (2009). Supervisão em habilidades sociais e seu papel na promoção deste repertório em estagiários de psicologia. Revista Brasileira de Terapias Cognitivas, 5(1), 18-34.

Bolsoni-Silva, A. T., Loureiro, S. R., Rosa, C. F., \& Oliveira, M. C. F. A. (2010). Caracterização das habilidades sociais de universitários. Contextos Clínicos, $3(1), 62-75$.

Bueno, J. M. H., Lemos, C. G., \& Tomé, F. A. M. F. (2004). Interesses profissionais de um grupo de estudantes de psicologia e suas relações com inteligência e personalidade. Psicologia em Estudo, 9(2), 271-278.

Bueno, J. M. H., Oliveira, S. M. S. S., \& Oliveira, J. C. S. (2001). Um estudo correlacional entre habilidades sociais e traços de personalidade. PsicoUSF, $6(1), 31-38$.

Caballo, V. E. (2003). Manual de avaliação e treinamento das habilidades sociais. São Paulo: Santos.

Caballo, V. E. (1996). O treinamento em habilidades sociais. In V. E. Caballo (Org.). Manual de técnicas de terapia e modificação do comportamento (pp. 3-42). São Paulo: Santos.

Campos, L. F. L., Silva-Filho, N. S., Campos, P. R., \& Rocha, R. L. (1996). Caracterização dos alunos de Psicologia da USF/Itatiba: características, opiniões e expectativas. PsicoUSF, 1(2), 57-82. 
Carmo, M. C. (2011). O papel dos afetos no processo de formação de psicólogos. Dissertação de Mestrado, Programa de Pós Graduação Stricto Sensu em Psicologia, Pontifícia Universidade Católica de Campinas, Campinas.

Carneiro, R. S., Falcone, E., Clarck, C., Prette, Z. D., \& Prette, A. D. (2007). Qualidade de vida, apoio social e depressão em idosos: relação com habilidades sociais. Psicologia: Reflexão e Crítica, 20(2), 229-237.

Cia, F., Pamplin, R. C. O., \& Del Prette, Z. A. P. (2006). Comunicação e participação pais-filhos: correlação com habilidades sociais e problemas de comportamento dos filhos. Paidéia, 16(35), 395-406.

Costa, C. C., Bastiani, M., Geyer, J. G., Calvetti, P. Ü., Muller, M. C., \& Moraes, M. L. A. (2008). Qualidade de vida e bem-estar espiritual em universitários de psicologia. Psicologia em Estudo, 13(2), 249-255.

Couto, G., Muniz, M., Vandenberghe, L., \& Van Hattum, A, C. (2008). Diferenças relacionadas ao sexo observadas no Checklist de Relações InterpessoaisRevisado. Avaliação Psicológica, 7(3), 347-357.

Couto, G., Van Hattum, A. C. F. M., Vandenberghe, L. M. A.. \& Benfica, E. (2005). Tradução, análise semântica e adaptação do Check List of Interpesonal Transactions-Revisado. Avaliação Psicológica, 4(1), 45-57.

Couto, G., Vandenberghe, L., Van Hattum, A. C., \& Campos, H. R (2006). Propriedades Psicométricas do Checklist de Relações Interpessoais Revisado. Psicologia Argumento, 24(47), 15-28.

Del Prette, Z. A. P. (1986). O papel social da Psicologia: reflexões sobre critérios e métodos para avaliar a relevância de intervenções na comunidade. Ciência e Cultura, 38(2).

Del Prette, A., Del Prette, Z. A. P, \& Castelo Branco, U. V. (1992). Competência social na formação do psicólogo. Paidéia, 2, 40-50.

Del Prette, Z. A. P., \& Del Prette, A. (1983). Análise de repertório assertivo em estudantes de Psicologia. Revista de Psicologia, 1, 15-24.

Del Prette, Z. A. P., \& Del Prette, A. (1999). Psicologia das habilidades sociais: terapia e educação. Petrópolis, RJ: Vozes.

Del Prette, Z. A. P. \& Del Prette, A. (2001a). Psicologia das relações interpessoais: vivências para o trabalho em grupo. Petrópolis, RJ: Vozes.

Del Prette, Z. A. P., \& Del Prette, A. (2001b). Manual do Inventário de Habilidades Sociais (IHS). São Paulo: Casa do Psicólogo. 
Del Prette, Z. A. P., Del Prette, A., Barreto, M. C. M., Bandeira, M., Rios-Saldaña, M. R., Ulian, A. L. A. O., Gerk-Carneiro, E., Falcone, E. M. O., \& Villa, M. B. (2004). Habilidades sociais de estudantes de Psicologia: um estudo multicêntrico. Psicologia Reflexão e Crítica, 17(3), 341-350.

Dickinson, D., Bellack, A. S., \& Gold, J. M. (2007). Social/communication skills, cognition, and vocational functioning in schizophrenia. Schizophrenia Bulletin, 33(5), 1213-1220.

Eisenberg, N., Fabes, R., Carlo, G., Peer, A. L., Swintzer, G., Karbons, M. \& Troyer, D. (1993). The relations of empathy-related emotions and maternal practices to children's comforting behavior. Journal of Experimental Child Development, 55, 131-150.

Fisher, G. A. (1996). Theoretical and methodological elaborations of the circumplex models of personality traits and emotions. In: R., Plutchick, $\mathrm{H}$. R., Comte (Orgs.). Circumplex models of personality and emotions (pp. 245-269).

Gomide, P. I. C., Salvo, C. G., Pinheiro, D. P. N., \& Sabbag, G. M. (2005). Correlação entre práticas educativas, depressão, stress e habilidades sociais. Psico-USF, 10(2), 169-178.

Kiesler, D. J. (1983). The 1982 Interpersonal Circle: A Taxonomy for Complementarity in Human Transactions. Psychological Review, 90, 185214.

Kiesler, D. J., Goldston, C. S., \& Schmidt, J. A. (1991). Manual for Check List of Interpersonal Transactions - Revised and Check List of Psychoterapy Transactions - Revised. Virginia: Virginia Commonwealth University.

Kliewer, W. (1991). Coping in middle clidhood: relations to competence, type a behavior, monitoring, blunting and locus of control. Developmental Psychology, 27(4), 689-697.

Lazarus, A. A. (1973). On assertive behavior: a brief note. Behavior Therapy, 4, 697-699.

Rathus, S. A. (1975). Principles and practices of assertive training: an eclectic overview. The Counseling Psychologist, 5, 9-20.

Riggio, R. E., Watring, K. P., \& Throckmorton, B. (1993). Social skills, social support, and psychosocial adjustment. Personality and Individual Differences, 15(3), 275-280. 
Wolpe, J. S. (1976). A prática da terapia comportamental. São Paulo: Brasiliense.

Contato: wawamt@hotmail.com, gleibercouto@yahoo.com.br, renatalimongi@yahoo.com.br

Recebido em: 10/02/2012

Revisado em: 18/04/2012

Aceito em: 19/06/2012 